\title{
Flipped Classroom: una experiencia para fortalecer el aprendizaje en Medicina Veterinaria
}

\author{
Alvaro Adriazola Uribe ${ }^{1}$ \\ ORCID: 0000-0002-5808-0598 \\ Georgina Duran Jimenez ${ }^{2}$ \\ ORCID: 0000-0001-6047-867X \\ Marcelo Flores Troncoso ${ }^{1}$ \\ ORCID: 000-0002-6673-0845
}

\section{Resumen}

Flipped Classroom (FC) es un modelo pedagógico, que utiliza las Tecnologías de la Información y la Comunicación (TIC), que consiste en intercambiar las actividades: lo que regularmente se veía en clase ahora se lleva para la casa y lo que se llevaba para la casa ahora se aborda en la clase. Es conocido por optimizar el tiempo del aula y por permitir un mayor desenvolvimiento de los estudiantes en colectividad. El modelo FC trae implícito el cambio de rol del profesor y el estudiante. El docente ya no es más quien da la cátedra, sino un mediador o guía que facilita las discusiones y las reflexiones, cede el control del aprendizaje brindando apoyo cuando el estudiante lo precisa. Mientras que el estudiante está más implicado, aprende a trabajar en equipo, desarrolla habilidades en la práctica y construye conocimiento colaborativamente. El objetivo de estudio fue analizar la interacción del FC en el trabajo colaborativo y construcción de conocimiento de estudiantes de Medicina Veterinaria (MV). La investigación se realizó a 44 estudiantes de MV, bajo la temática Clasificación de Fracturas en Pequeños Animales. Se distribuyeron en dos grupos: aula tradicional y FC. Los resultados cuantitativos se obtuvieron con el software SPSS, mientras que los datos cualitativos se analizaron por categorías. El FC fue un modelo que construyó conocimientos y favoreció el trabajo colaborativo entre los estudiantes en mayor medida que el aula tradicional.

\section{Palabras clave}

Educación - Conocimiento - TIC - Sala de aula invertida - Metodología de enseñanza.

1- Universidad Católica de Temuco, Facultad de Recursos Naturales, Departamento de Ciencias Veterinarias, Temuco, Chile. Contactos: alvaro.adriazola.uribe@gmail.com; mflores@uct.cl.

2-Universidad Federal de Bahía, Salvador, Brasil.Contacto: duran_gi@hotmail.com 


\section{Flipped classroom: an experience to strengthen learning in Veterinary Medicine}

\section{Abstract}

Flipped Classroom (FC) is a pedagogical model, which uses Information and Communication Technologies (ICT) to interchange activities: what was regularly seen in class is then taken home and what was taken home is then addressed in class. It is known for optimizing classroom time and for allowing more development students in group work. The FC model implies a role change in the teacher-student relationship. The teacher is no longer the one who directs the class, but a mediator or guide who facilitates discussions and reflections, so learning is in control by providing support when the student needs it. When students get involved, they learn to work in groups, develop skills in hands-on and collaborative knowledge building. The objective of the study was to analyze the interaction through FC in the collaborative work and knowledge construction among Veterinary Medicine (VM) students. The study was conducted with 44 VM students, the topic was Classification of Fractures in Small Animals. They were divided in two groups: traditional classroom and FC. Quantitative results were obtained with SPSS software, while qualitative data were analyzed by categories. The FC was a model that built knowledge and increased collaborative work among students to a greater extent than the traditional classroom.

\section{Keywords}

Education - Knowledge - ICT - Flipped classroom - Teaching methodology.

\section{Introducción}

A lo largo de los años, la humanidad ha experimentado variaciones políticas y económicas que han transformado los modos de vida. Estos cambios fueron marcados en el tiempo por tres grandes olas, la revolución agrícola, la revolución industrial y la sociedad postindustrial (TOFFLER, 1980). Esta última ola se distingue por sus grandes avances y sus nuevas formas de relacionarse, los cuales surgieron de una revolución digital, de información y comunicación que no solo envolvió al sector político y al económico, sino que paulatinamente ganó terreno en todas las esferas de la sociedad. Manuel Castells (2000) se refiere a esta inédita forma de organización social interconectada como la nueva sociedad red, la cual, ha extendido y expandido el uso cotidiano de las tecnologías de la información y la comunicación (TIC) hasta nuestros días.

\section{De la educación tradicional a la educación con el uso de TIC}

A pesar del frecuente empleo de las TIC por la sociedad y de la gran evolución que este campo ha tenido en los últimos años, sus usos y su apropiación en las instituciones 
educativas han sido mínimos y paulatinos. Son diferentes los motivos por los que algunos profesores prefieren no incursionar en la utilización de tecnologías y de ambientes virtuales, pero se relaciona principalmente al hecho de que la mayoría de ellos hicieron una transición paulatina de lo análogo a lo digital dentro de su vida personal, mientras que su incursión en las instituciones educativas fue con poca previsión y sin procesos de formación continuos. Lo recomendable dentro del campo de las TIC es implicar a los profesores a vivir experiencias, producir y socializar con estos ambientes, porque solo esta convivencia-formación puede traer apropiación de forma no lineal (SAMPAIO-S; BONILLA, 2014). De otro modo, se puede provocar negación al uso de las TIC o desconocimiento absoluto de cómo apropiarse crítica y dinámicamente de ellas (como una fortaleza), en vez de que como una distracción.

Un problema que se detecta en dejar a las TIC fuera del proceso de enseñanza-aprendizaje o de utilizarlas técnicamente como simples herramientas que sustituyen los antiguos materiales (pizarrón, tiza, pliegos de papel, etc.), es que el profesor no puede construir un diálogo con sus estudiantes, porque desconoce el lenguaje de las nuevas generaciones, que crecen y se desarrollan con las TIC (BONILLA, 2011). Esto provoca una formación insuficiente para desenvolverse en un mundo acelerado para y por la tecnología digital, en este sentido, “[...] no están siendo formados los profesionales para el mundo que se está construyendo al tiempo que las mudanzas ocurren de forma veloz en grandes dimensiones" (PRETTO, 2013, p. 123, traducción nuestra).

Por otro lado, una de las potencialidades que permite la implementación de las TIC en la educación es que favorece el cambio de rol entre el profesor y el estudiante. Hasta hace un par de décadas el protagonista del aula era el profesor, un repetidor que daba clases homogéneas, disciplinares, magistrales de instrucción directa y dicho profesor repetidor enseñaba al estudiante (GALEFFI, 2017). Con los cambios en las relaciones sociales y la inmersión de las TIC a la escuela surgieron diferentes métodos de enseñanza y modelos educativos que permitieron mayor autonomía y protagonismo de su propio aprendizaje al estudiante, quien tal como lo planteó Vigotsky (1978 apud WERTSCH, 1988) años atrás, con orientación de su profesor y trabajo colaborativo con sus iguales, es capaz de construir su propio conocimiento.

Pese a que existen bloques de métodos didácticos de acuerdo al enfoque que estos utilizan, Miguel Díaz (2005) distingue tres: el enfoque didáctico para la individualización, el enfoque de la socialización didáctica y el enfoque globalizado. Cada uno de ellos tiene diferentes metodologías que traen al aula actividades centradas en el estudiante, pero por lo general no son incluyentes entre sí y cada uno es reduccionista respecto a su planteamiento original, ya sea individualista, social y/o global. Sin embargo, los desafíos que exige la contemporaneidad a la educación precisan de métodos didácticos menos disyuntivos y/o reduccionistas, que sean capaces de potencializar el aprendizaje integral del ser humano y, a su vez, hacer frente de las demandas actuales de los contextos. Así se impulsa la inserción de lo que ahora se conoce como bricolaje en la educación, que aunque es un concepto que todavía está en construcción, se aborda con la diversidad de teorías, métodos, modelos y/o materiales adaptados en un contexto para la construcción de la investigación y/o la educación (KINCHELOE, 2004).

En este sentido y debido a sus características maleables, se puede decir que tanto las TIC como el bricolaje se potencializan de forma inherente. Sus implementaciones 
permiten adaptaciones para usos en actividades dentro y fuera del aula, favoreciendo diversos métodos, modelos y/o materiales que pueden fortalecer el proceso de enseñanzaaprendizaje, además de la autonomía y la creatividad de los estudiantes. En consecuencia, se perfilan como una respuesta poderosa al reto de la educación contemporánea, desde “[...] la articulación colectiva, colaborativa, abierta, en un momento en que todavía estamos presos a modelos cerrados e individualistas” (BONILLA; PRETT0, 2015, p. 16, traducción nuestra), posibilitando un proceso de enseñanza-aprendizaje dinámico, a partir de la construcción crítica, creativa y colaborativa de conocimiento.

Un modelo pedagógico que se sustenta en las TIC y que favorece el bricolaje es el Flipped Classroom o la clase al revés, que consiste en intercambiar las actividades del aula: lo que regularmente se veía en clase ahora se lleva para la casa y lo que se llevaba para la casa ahora se aborda en la clase. Este modelo, aunque no es el único efectivo para la utilización de TIC y favorecimiento del bricolaje, se distingue porque su diseño optimiza el tiempo en el aula, posibilitando el incremento de actividades que los profesores pueden trabajar en el aula y otorga autonomía al estudiante, permitiendo un mayor desenvolvimiento en colectividad (y al mismo tiempo personal) entre los estudiantes.

\section{Metodología del modelo Flipped Classroom}

En su aplicación se distinguen tres grandes fases, la primera es la interacción en casa del estudiante con un video educativo y las lecturas complementarias opcionales, la segunda es la discusión y el debate de los estudiantes mediante la orientación del profesor, que comienza con las preguntas o dudas surgidas del material trabajado en casa y la tercera es el reforzamiento por grupos de lo aprendido mediante prácticas y/o actividades. Los creadores del Flipped Classroom Bergmann y Sams (2012), así como sólo Bergmann (2014) y Hirsch (2014), narran cómo hacer una buena praxis en el uso de este modelo y comparten consejos respecto a cómo superar ciertas dificultades con las que ellos se han encontrado a lo largo de su experiencia de trabajo con este modelo.

Respecto a las características del video, los autores señalan que es mejor que sea corto, con una duración de hasta 15 minutos como máximo, porque esto mantiene de mejor manera la atención de los estudiantes, incluso se dice que es preferible crear dos videos cortos que uno mayor a 15 minutos. No hay que preocuparse mucho por las cuestiones técnicas, en el sentido de que es un video educativo y no un documental, por lo que puede ser un material sencillo, pero sin descuidar detalles como la buena iluminación de la pantalla de la computadora o el volumen. Si el profesor va a aparecer en el video, también debe tomar en cuenta que haya una buena iluminación del lugar y evitar los ruidos y/o las distracciones de fondo. El video puede ser de autoría personal, existen softwares libres que auxilian de forma sencilla a su realización (Screencast u OBS Studio) o se pueden editar videos previamente diseñados, esto también se puede hacer con el apoyo de softwares libres (Openshot o Avidemux). Cuando se trate de la edición de videos realizados por otros, hay que asegurarse de respetar los derechos de autor y las licencias del material, si es que tuviesen.

Dentro de las cuestiones académicas, la planificación del video debe ser cuidadosa respecto a los objetivos de aprendizaje que se quieran alcanzar en el tema. Si se quiere tener 
seguridad de que los estudiantes entraron al video, colocar notas, preguntas abiertas, de opción múltiple y/o controlar cuestiones como cuánto tiempo lo vieron, si lo adelantaron o etc., existen softwares gratuitos que coadyuvan a contabilizarlo (Videonot, eduCanon o Playposit). Estos recursos permiten contrarrestar desventajas como el hecho de que el profesor no esté físicamente para realizar preguntas en tiempo real e, incluso, algunos de estos softwares permiten la integración de otros ambientes gratuitos (Google Drive) para almacenar archivos de preguntas o problemas más extensos.

Antes de comenzar a usar el Flipped Classroom, se recomienda que el profesor explique a los estudiantes la forma en que se llevará a cabo la nueva dinámica de clase y posteriormente se les muestre cómo interactuar con el video. Como mencionan Bergmann y Sams (2012), el estudiante no sólo va a ver, sino a interactuar con el video, por eso es importante que no tenga otras distracciones tecnológicas alrededor, si es un video sencillo sin la inserción de otros recursos, deben saber dar pausa si necesitan pensar una idea, para anotar los puntos clave, las dudas y/o preguntas que tengan en determinado fragmento. Y si tiene la inserción de recursos, como preguntas o problemas que el profesor haya planteado en el video, también se debe indicar un momento para pausarlo, o bien el estudiante puede repetirlo las veces que fuera necesario.

Una vez finalizada la edición del material, es importante que el profesor lo asista en su totalidad para comprobar que todo funciona correctamente. También, antes de encomendar la tarea, el profesor debe asegurarse que los estudiantes tengan libre acceso al video. Si se compartirá un link en algún sitio, es recomendable comprobar el acceso o si se subió a internet, se aconseja revisar que se visualice íntegro desde un dispositivo diferente al que se ocupó para compartirlo. Los mismos criterios de verificación se aplican si se encargan lecturas complementarias.

Es primordial que el profesor se mantenga firme en la posición de no reproducir el video dentro del aula, para ir formando la cultura de funcionamiento de la nueva dinámica. Generalmente la clase comienza con la formación de pequeños equipos y se parte a la discusión del video y las lecturas. Después de responder las preguntas o dudas entre los estudiantes, donde el profesor sólo interviene si fuese necesario, se pasa a la práctica o actividad. Dependiendo de la materia pueden hacerse prácticas en el laboratorio, resolución de problemas, estudios de casos, cuestionarios, pruebas, etc., siempre en pequeños equipos, esto con la finalidad de que colaborativamente cada equipo consiga analizar, evaluar y retroalimentarse entre ellos, para construir así su propio conocimiento a partir de sus experiencias y sus aprendizajes individuales anteriores.

Las actividades o prácticas pueden plantearse con el apoyo de softwares gratuitos (Proprofs, Socrative, Thatquiz, Questionpro o Google Drive) o en la plataforma educativa de la institución educativa (si es que trabaja con alguna). Los nombres que se mencionan a lo largo del texto son sólo algunos ejemplos, pero existen más softwares y ambientes virtuales que permiten infinidad de alternativas de actividades, con diversos recursos y estrategias para explorar. Se use el que se use, lo fundamental es cuidar los objetivos de aprendizaje de las prácticas o actividades, así como intentar que con ellas se alcancen niveles elevados de razonamientos y pensamientos críticos y poner a prueba habilidades, donde se tenga que hacer uso de los conocimientos que ya se tienen dentro de prácticas solidarias y colaborativas. 
Como se puede observar, el Flipped Classroom tiene implícita la ruptura de los roles tradicionales del profesor y del estudiante. El docente ya no es más quien da la cátedra, sino un orientador o mediador que facilita las discusiones y las reflexiones, cede el control del proceso de enseñanza-aprendizaje y brinda apoyo sólo cuando el estudiante lo necesita. Por otro lado, el estudiante está más implicado en las actividades y los contenidos, aprende a trabajar en colectividad, desarrolla habilidades en las prácticas entre sus iguales y construye conocimientos colaborativamente.

Por sus características, este modelo pedagógico se presenta ideal para las disciplinas del área de ciencias, donde los profesores muestran mayor dificultad para introducir el uso de las TIC en el aula, principalmente por el miedo a no cumplir con los objetivos de aprendizaje. Sin embargo, una buena praxis del Flipped Classroom cuida en todas sus fases de estos objetivos; y en el tiempo en el aula, con los estudios de caso, problemas y/o prácticas -efectivos también para las ciencias- consigue llevar a la realidad el aprendizaje, reforzarlo y desarrollar habilidades específicas del perfil en el que se desenvuelvan.

Por esto, se planteó la realización de un estudio en el área de Medicina Veterinaria, que busca esa implicación de lo riguroso, específico y complejo del área al modelo Flipped Classroom, al mismo tiempo que explora la interacción de los profesores y los estudiantes con la implementación y el uso de las TIC en su proceso de enseñanza-aprendizaje y la construcción de conocimientos en colectividad.

\section{Material y métodos}

La investigación se llevó a cabo en 2014, en una Escuela de Medicina Veterinaria perteneciente a la Facultad de Recursos Naturales de una Universidad en Chile. La población consistió en 44 estudiantes de la asignatura de Internado II, pertenecientes al $5^{\circ}$ año de la carrera. Así como los dos profesores encargados de impartir esa cátedra, quienes tuvieron la iniciativa de testar el uso del modelo Flipped Classroom en su aula con horas teórico - prácticas. Por lo tanto, el criterio de selección del grupo fue por conveniencia.

El objetivo general de la investigación se centra en analizar comprensivamente el trabajo colaborativo y la construcción de conocimiento de los estudiantes en una clase con el modelo Flipped Classroom y su respectivo uso de TIC. A partir de este se desprenden tres objetivos específicos:

- Analizar el desenvolvimiento de los estudiantes en una clase con el modelo Flipped Classroom y en otra con el modelo tradicional magistral.

- Analizar comprensivamente la relación profesores - estudiantes en una clase con el modelo Flipped Classroom y en una con el modelo tradicional magistral.

- Valorar la construcción de conocimiento y el trabajo colaborativo de los estudiantes con el modelo Flipped Classroom y el modelo tradicional magistral.

Como se objetivó analizar comprensivamente el trabajo colaborativo y la construcción de conocimiento de los estudiantes utilizando el Flipped Classroom, se quisieron observar todos los escenarios posibles en la construcción de conocimiento de los mismos, por eso se decidió utilizar un diseño mixto de estrategia de triangulación concomitante (CRESWELL, 
2007), porque permitió la producción de datos cuantitativos y cualitativos al mismo nivel y simultáneamente. Para el levantamiento de los datos cuantitativos se usó un diseño de tipo cuasi experimental sólo post intervención, simple y simultáneo (LEÓN; MONTERO, 2003), porque permitió analizar ambos formatos de clase (magistral y Flipped Classroom), mientras que para los datos cualitativos, se empleó la observación participante (FLICK, 2009), porque permitió dinamismo a los profesores, una vez que al mismo tiempo que dieron su aula, produjeron sus percepciones.

En la metodología se decidió dividir a los participantes en dos grupos, determinado por el orden de la lista del curso. La primera mitad formó el grupo de cuasi control y la segunda mitad el grupo experimental. Por cuestiones personales ocho estudiantes no consiguieron llegar el día de la clase magistral ${ }^{3}$, por lo que el grupo de cuasi control se formó con dieciocho estudiantes (nueve hombres y nueve mujeres) y el grupo experimental con veintiséis (diez hombres y dieciséis mujeres) (Tabla 1). Cabe señalar que la variable nominal sexo, aparece únicamente con sentido representativo en ambos grupos, no se utiliza para comparación entre ellos o entre los grupos.

Tabla 1- Descripción de la muestra y la división de grupos

\begin{tabular}{|c|c|c|c|c|c|}
\hline & & & \multicolumn{2}{|c|}{ Sexo } & \multirow[t]{2}{*}{ Total } \\
\hline & & & Hombre & Mujer & \\
\hline \multirow[t]{12}{*}{ Clase } & $\begin{array}{c}\text { Magistral } \\
\text { Cuasi Control }\end{array}$ & Recuento & 9 & 9 & 18 \\
\hline & & $\%$ de Clase & $50.0 \%$ & $50.0 \%$ & $100.0 \%$ \\
\hline & & $\%$ de Sexo & $47.4 \%$ & $36.0 \%$ & $40.9 \%$ \\
\hline & & $\%$ del total & $20.5 \%$ & $20.5 \%$ & $40.9 \%$ \\
\hline & $\begin{array}{c}\text { Flipped Classroom } \\
\text { Experimental }\end{array}$ & Recuento & 10 & 16 & 26 \\
\hline & & $\%$ de Clase & $38.5 \%$ & $61.5 \%$ & $100.0 \%$ \\
\hline & & $\%$ de Sexo & $52.6 \%$ & $64.0 \%$ & $59.1 \%$ \\
\hline & & $\%$ del total & $22.7 \%$ & $36.4 \%$ & $59.1 \%$ \\
\hline & \multirow[t]{4}{*}{ Total } & Recuento & 19 & 25 & 44 \\
\hline & & $\%$ de Clase & $43.2 \%$ & $56.8 \%$ & $100.0 \%$ \\
\hline & & $\%$ de Sexo & $100.0 \%$ & $100.0 \%$ & $100.0 \%$ \\
\hline & & $\%$ del total & $43.2 \%$ & $56.8 \%$ & $100.0 \%$ \\
\hline
\end{tabular}

Fuente: Elaboración propia.

Para la intervención del Flipped Classroom, este último grupo se dividió en 5 equipos asignados por orden de llegada al aula, cuatro equipos tuvieron cinco participantes y uno quedó con seis participantes. En todo momento de las clases, los dos grupos contaron con sus dos profesores que les impartían la materia.

3- Se utiliza el término clase magistral para referirse a la impartición de la cátedra tradicional, como regularmente los profesores trabajaban sus aulas: ellos exponiendo un tema y los estudiantes interviniendo si les surgía alguna duda o una pregunta durante la explicación. 
La secuencia didáctica comenzó por informar a los estudiantes, antes de que se llevaran a cabo las clases, sobre qué era el modelo Flipped Classroom y lo que posteriormente se quería testear con él en el aula. Una vez que se obtuvo el consentimiento de todos los participantes, se prosiguió a agendar las sesiones y a explicar la metodología que se utilizaría para las mismas.

Se estudió el tópico científico de Clasificación de Fracturas en Pequeños Animales para la planeación de las clases - de hora y media cada una- que se impartirían a los dos grupos. Al grupo de cuasi control se le pidió que se presentara a la clase magistral, para la que se elaboró una presentación en diapositivas, como regularmente se trabajaba en el aula tradicional y todo transcurrió con total normalidad y cotidianidad. Posteriormente, como material de estudio fueron enviadas dos lecturas a los participantes de este grupo, incluyendo a los ocho estudiantes que por motivos personales no pudieron asistir a la clase.

Para el grupo experimental se empleó la misma presentación en diapositivas que se usó con el grupo de cuasi control, pero con la ayuda de un software se grabó la voz de uno de los profesores. Mientras el profesor explicaba simplificadamente -pero cuidando los objetivos de aprendizaje- la presentación era proyectada en la pantalla de su computadora y manipulada por él mismo al ritmo de su explicación, así se elaboró un video de 14 minutos. Este video (previamente se explicó a los estudiantes cómo interactuar con él) y las mismas dos lecturas que se mandaron al grupo de cuasi control, fueron enviadas al grupo experimental como reforzadoras del tema, un día antes de que se llevara a cabo su clase. Se aprovechó para mencionarles que aquellos que tuvieran tableta o computadora y quisieran llevarla a la clase, podian hacerlo.

El día de la clase del grupo experimental se formó a los participantes en grupos. Uno de los profesores abrió el diálogo preguntando si había dudas o preguntas del material visto en casa, los estudiantes hicieron algunas preguntas y el profesor auxilió a responder cuestionando a los mismos estudiantes quién podía responder, esto no llevó más de 10 minutos.

Mientras esa primera fase de la clase acontecía, el otro profesor enviaba a los estudiantes el formulario que sería el material para el aula. Este se realizó en Google Drive, debido a que después de ser respondido, recopila los datos proporcionados por los participantes, siendo así el instrumento de recolección de datos cuantitativos. Dicho formulario constaba de dieciocho ítems de opción múltiple. Aunque las preguntas no estaban agrupadas, se distinguían tres dimensiones del tópico científico: Reconocimiento de Anatomía Radiográfica (RAR) con dos ítems, Clasificación Radiográfica de Fracturas (CFF) con trece ítems e Integradoras de Conocimiento en Casos Clínicos (ICCC) con tres ítems.

Para la construcción del formulario se tuvo en cuenta el atendimiento de los objetivos de aprendizaje del tópico científico, los objetivos de la investigación y la consulta de expertos en el modelo Flipped Classroom, así como su validación a través de criterio de expertos en el modelo y en cirugía de pequeños animales.

La actividad duró los 80 minutos restantes de la clase y pese a que todos los integrantes del equipo podían entrar a ver las preguntas, se les pidió sólo un formulario respondido por equipo. Así, los participantes de cada equipo tenían que debatir sobre el video y las lecturas y colaborativamente evaluar las preguntas y retroalimentarse, para construir y decidir sus respuestas. Al inicio de la actividad se les ayudó con dudas técnicas sobre el manejo de los equipos de la universidad (tabletas que les fueron proporcionadas 
a aquellos que no llevaron su propio equipo), así como con algunas dudas que les surgían sobre el tema mientras contestaban su material. Cabe señalar que si la pregunta que hacían los participantes influía o implícitamente dejaba clara la respuesta de algo que estuviese en el material, los profesores no la respondían, para no intervenir en los resultados.

Simultáneamente, pero de manera individual y no presencial, el grupo de cuasi control también tuvo acceso al formulario. Se dieron aproximadamente noventa minutos para resolverlo, así que fue abierto desde que comenzó la clase con el grupo experimental y cuando esta terminó, el envío de respuestas también fue cerrado. No se afectó el tiempo personal de nadie porque ese era el horario de su clase habitual.

La observación participante se llevó a cabo directamente cuando se desarrollaban las clases de ambos grupos y posteriormente se realizó una conversación con los dos profesores presentes para contrastar perspectivas, así como reflexiones generales y específicas de la experiencia, que atendieran los objetivos de la investigación.

\section{Resultados y discusión}

Los resultados cuantitativos se obtuvieron con ayuda del software SPSS versión 15.0, mientras que los datos cualitativos se analizaron por categorías. Posteriormente se triangularon entre ellos y con la teoría que sustenta la investigación.

Respecto a los dos tipos de metodologías usadas en el aula, los datos se mostraron por encima de la media, favorable para el modelo Flipped Classroom en contraste con la clase magistral (Gráfica 1).

Gráfica 1- Análisis general de la Clase Magistral y la Flipped Classroom

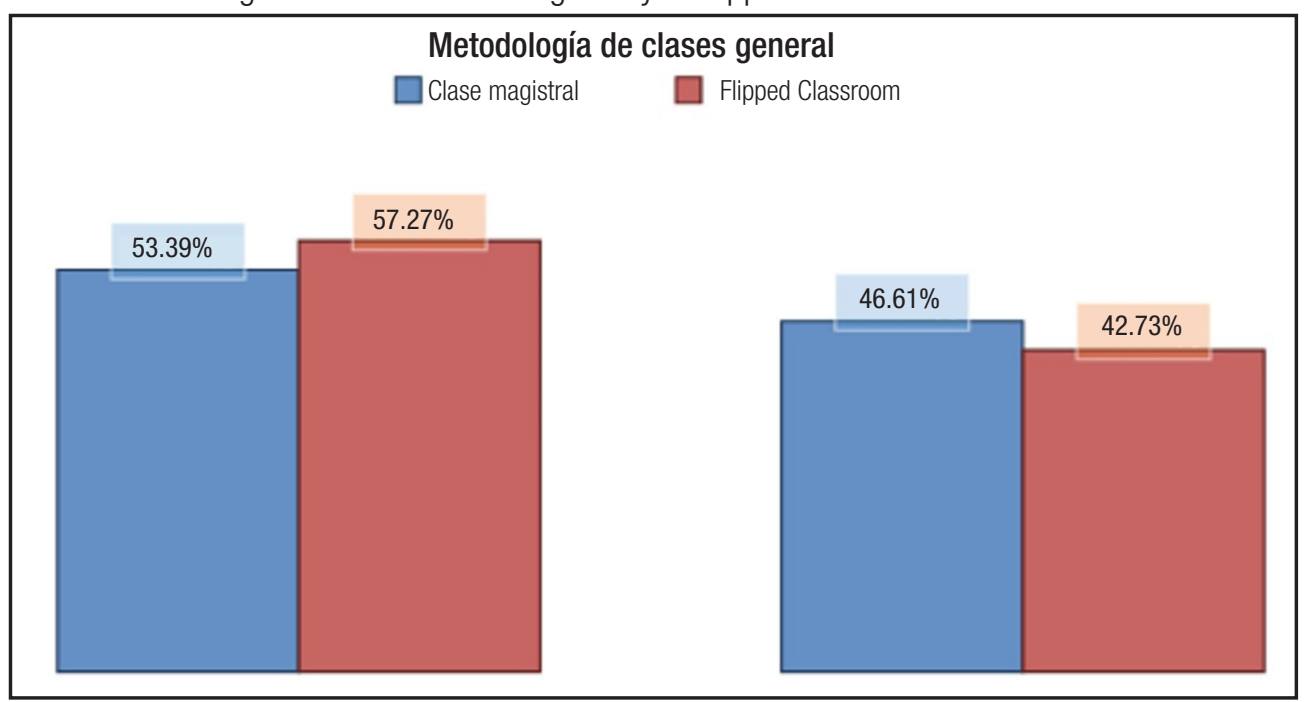

Fuente: Elaboración propia.

De acuerdo a las observaciones de los profesores, con el modelo Flipped Classroom, el uso del tiempo en el aula es más provechoso. Aunque a primera instancia les pareció 
más complicado trabajar con esta metodología -por la elaboración del video y del material para la clase- se convencieron que el uso de la clase magistral es un tema cultural y de costumbre, así como de desconocimiento en el no uso de nuevos modelos o metodologías para las aulas.

En este sentido, a pesar de no ser expertos ni nativos de las TIC, los profesores no mostraron mayor problema en la construcción del vídeo y del material, al igual que en la asesoría de los estudiantes con los aparatos en el aula. Por lo cual, se concuerda con Bergmann y Sams (2012) en que para que una pedagogía con tecnología sea efectiva, la pedagogía siempre debe conducir a la tecnología y nunca al revés.

Respecto a las percepciones personales, los profesores dijeron sentirse cómodos en ambas clases, pero valoraron como mayormente ventajosa la de Flipped Classroom, porque les permitió dejar de ser el centro de atención, pasando a ser un apoyo para que los estudiantes reforzaran conocimientos, construyeran conocimientos teórico/prácticos a su ritmo y a la vez aprendieran a trabajar en colectividad.

En los estudiantes que participaron del Flipped Classroom se pudo apreciar una confusión respecto a si eso que estaban haciendo era la clase o cuándo iba a comenzar la cátedra de los profesores, a la que estaban habituados. Durante la actividad los estudiantes se notaron interesados y colaborativos en el diálogo con sus compañeros de equipo. Mientras que en la clase magistral los participantes se comportaron como en cualquier otra clase, posteriormente al hacer la reflexión de ambas clases, la formalidad de la magistral consigue ser una barrera para el desarrollo y la interacción de los estudiantes.

De acuerdo con las observaciones de los profesores, debido al abordaje y la dinámica de la clase, los participantes de la Flipped Classroom se observaron más animados a preguntar y a participar, situación que no ocurre regularmente en la clase magistral, donde generalmente existe poca o nula participación, siendo prácticamente los mismos quienes preguntan o responden. En este grupo, los profesores también percibieron que las mujeres se mostraron más participativas de lo que regularmente son.

Respecto al uso de las TIC y al funcionamiento de los materiales virtuales, los estudiantes del grupo Flipped Classroom no tuvieron problemas mayores. En el aula algunos prefirieron trabajar con su equipo personal y otros con las tabletas de la universidad (los profesores ayudaron en algunas dudas técnicas de funcionamiento). Esto comprueba que los "[...] procesos formativos continuos, que vayan más allá de la dimensión meramente operativa de las máquinas digitales y que sean oportunos para la comprensión y la inmersión en los ambientes en red" (SAMPAIO; BONILLA, 2014, p. 26, traducción nuestra), son necesarios para apropiarse de modelos de aula que requieran ambientes digitales, porque dudas de la cultura digital, desde básicas hasta las más complejas, siempre se van a presentar.

Con relación a las clases, entre los dos grupos existieron algunas diferencias significativas (Gráfica 2). Cabe señalar que las dimensiones RAR (Reconocimiento de Anatomía Radiográfica), CFF (Clasificación Radiográfica de Fracturas) e ICCC (Integradoras de Conocimiento en Casos Clínicos) del formulario, se estructuraron de acuerdo a una dificultad progresiva. La dimensión CFF requería de conocimientos específicos, pensamiento racional y crítico; mientras que la ICCC consideraba lo mismo más conocimiento general extrapolado a casos reales. De acuerdo a los resultados, el modelo Flipped Classroom favoreció la dimensión CFF mostrando diferenciación porcentual ante la clase magistral. 
Gráfica 2- Análisis del Flipped Classroom ante la Clase Magistral

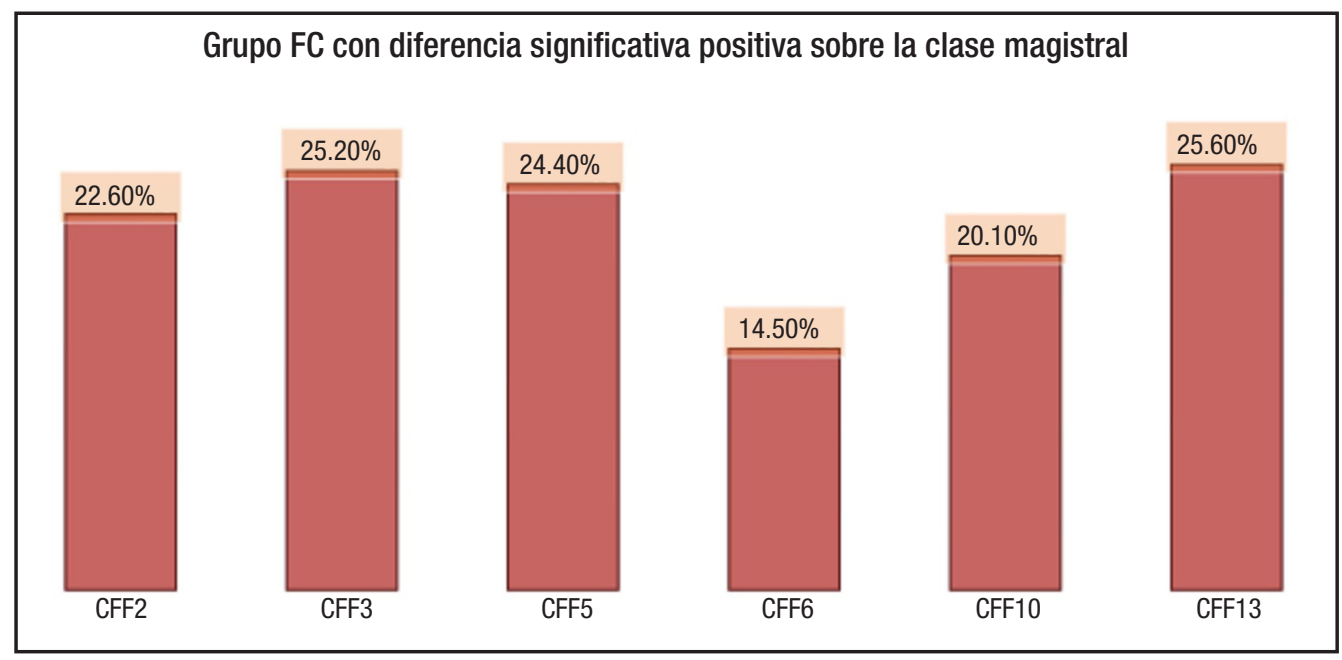

Fuente: Elaboración propia.

Con la observación, tanto para los resultados de la Gráfica 2 como para los de la Gráfica 4, se percibió que el diálogo que permite la dinámica del Flipped Classroom, así como el razonamiento crítico y la reflexión, ambos en colectividad, fueron los responsables de esas diferencias significativas. El debatir las ideas en conjunto puede conducir a una mejor respuesta que el hacerlo de forma individual.

Para determinar la construcción de conocimiento de los participantes en las dos clases, se hizo un análisis de lo contestado correcta e incorrectamente en el formulario. El resultado en general no es determinante (Gráfica 3), del 100\% de los estudiantes que contestaron correctamente el 59.09\% se localizó en el modelo Flipped Classroom. Sin embargo, de las respuestas incorrectas, también el mayor número se ubicó en ese grupo, con 57.01\% de errores.

Gráfica 3- Análisis del conocimiento general de los participantes

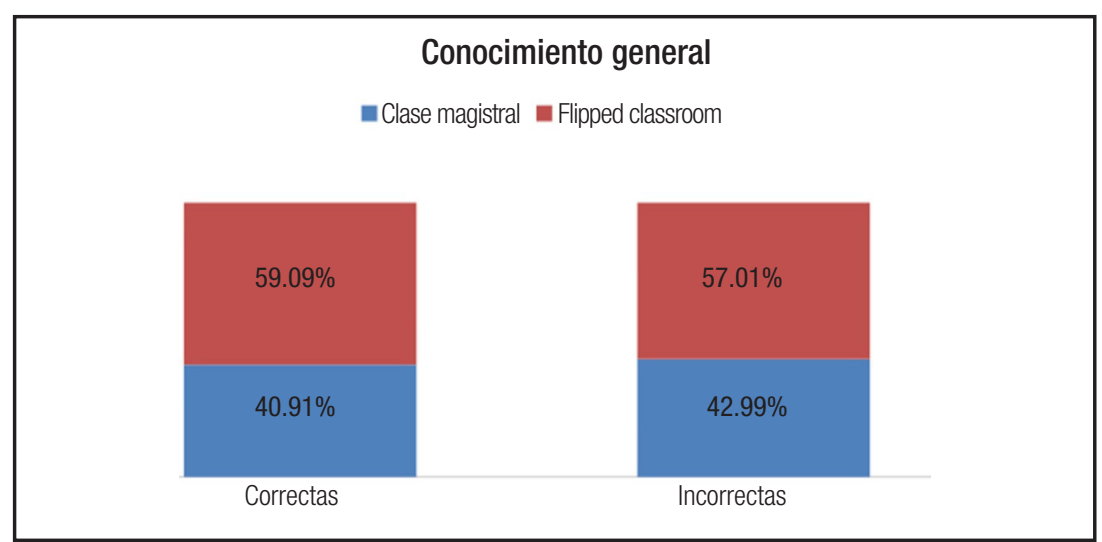

Fuente: Elaboración propia. 
Se cree que al ser mayor el desenvolvimiento de los participantes en la Flipped Classroom, consiguieron construir y retener mejor el aprendizaje. Sin embargo, es importante señalar que al ser un modelo de trabajo colectivo, las percepciones de los estudiantes para los otros estudiantes de su mismo equipo también pueden influenciar al decidir las respuestas, aunque todos debatan por igual, el diálogo de alguien puede ser más convincente e influenciar para ser tomado en cuenta aunque esté mal, sobre el de alguien que esté bien pero sea menos persuasivo.

En este sentido, la retroalimentación de las actividades (también en colectividad) por parte de los profesores es muy importante, para reforzar el aprendizaje o aprender de los errores y también para que, en particular en este modelo, con el uso frecuente se cultive la valoración de todas las opiniones por igual. Sin embargo, discutiendo aquí el rol de los estudiantes, se concuerda con la investigación de Davies, Dean y Ball (2013), aunque ellos tenían tres grupos (tradicional, independiente con simulación y Flipped Classroom), su grupo Flipped Classroom también mostró mayor adquisición de conocimiento. Además, al igual que en este estudio, su grupo Flipped Classroom manifestó ligeramente a su favor actitudes positivas, en transmisión de contenido y de conocimiento.

Volviendo al 100\% de respuestas correctas, algunos Ítems detectaron diferencias significativas (Gráfico 4). Nuevamente se localizaron en la dimensión CFF favoreciendo al modelo Flipped Classroom sobre la clase magistral.

Gráfica 4- Análisis de ítems correctos con diferencia significativa entre clases

\section{Conocimiento general}

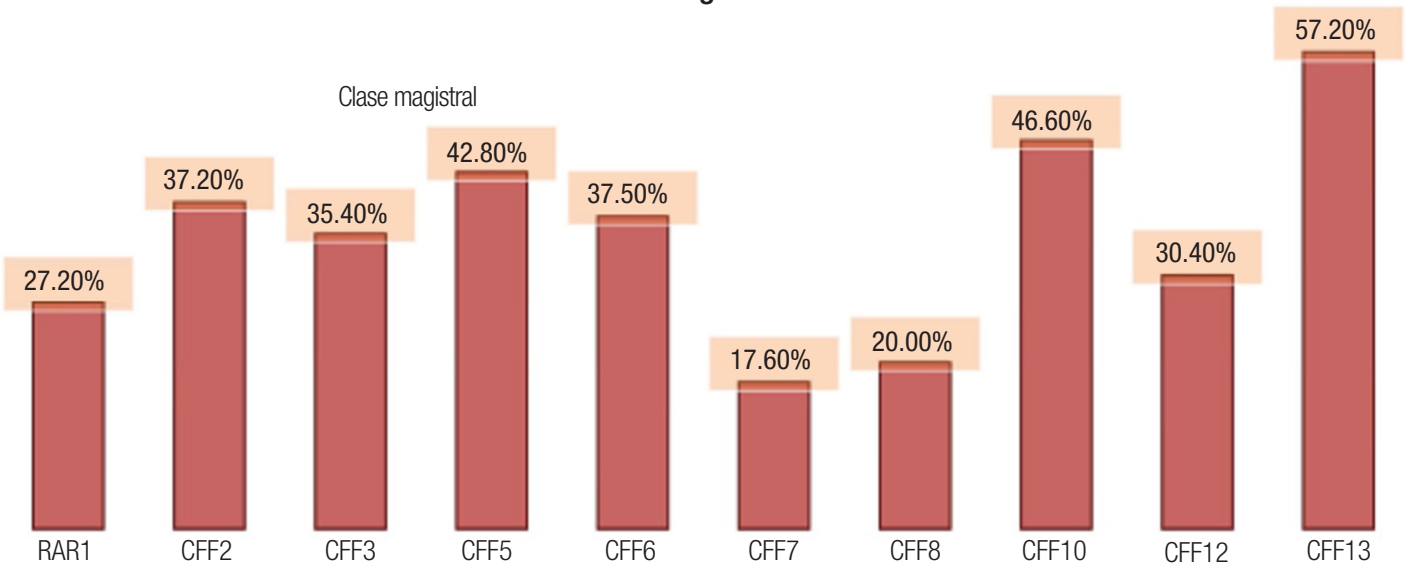

Fuente: Elaboración propia.

En el transcurso de la clase Flipped Classroom, en todos los equipos fue posible escuchar conversaciones que aparte de evidenciar conocimientos específicos, rescataban conocimientos de otras materias. Esta integración de conocimientos traídos a la actividad por diferentes estudiantes para ayudarse a responder correctamente, principalmente para los estudios de casos y casos clínicos, fue considerada como sumamente enriquecedora, 
pues no solamente compete este tema de esta disciplina, sino que es posible desenvolver lo aprendido en la carrera en general.

Los profesores señalaron esto como otra ventaja de la dinámica del Flipped Classroom para los estudiantes, que puede reforzar conocimiento a través de la construcción de nuevo conocimiento en colectividad. Como se observa, al igual que en el estudio de Cartón y otros autores (2015), la experiencia para los profesores ha sido positiva. Ambos coincidieron en el pensamiento de que el modelo es útil para los estudiantes por la aplicación de contenidos teóricos-prácticos, así como el fortalecimiento del análisis y del trabajo en grupo.

A su vez, se comprobó lo que señalan Bergmann y Sams (2012), que en el modelo magistral o tradicional los estudiantes que ponían más atención a los profesores y participaban eran siempre los mejores, mientras que con el Flipped Classroom los profesores distribuyen su atención entre todos los estudiantes, incluidos aquellos que en la clase magistral solo escuchan pasivamente, porque en la actividad de grupo sí participan y cuando necesitan ayuda preguntan con menos formalidad.

Por último, a diferencia del estudio de Mason, Shuman y Cook (2013), los estudiantes no se mostraron inicialmente frustrados con la clase Flipped Classroom, sólo se manifestaron confundidos con el funcionamiento del aula y hasta cierto punto, no creían que el trabajo en equipos fuera toda su clase, sintiendo extraño que sus profesores no estuvieran dando su cátedra de manera tradicional.

\section{Conclusiones}

En general se puede afirmar que esta experiencia fue satisfactoria, no obstante, es necesario testar un mayor número de veces y a lo largo de un curso completo, para obtener resultados de mayor envergadura.

En respuesta a los objetivos de la investigación, para el análisis comprensivo del Flipped Classroom, se concluyó que la construcción de conocimiento de los estudiantes va a depender de su trabajo individual en casa con el material, pero principalmente de la interacción colaborativa en equipo, donde la retroalimentación surge para enriquecer colectivamente.

En el desarrollo de los estudiantes con el modelo Flipped Classroom, se rescata el estímulo de su pensamiento crítico, discusiones y reflexiones al ritmo y estilo de cada uno. Aunque al principio desconocieron el formato de la clase, cuando notaron que se rompió esa formalidad de un aula magistral, se consiguió la participación de todos.

$\mathrm{Al}$ analizar comprensivamente la relación profesores-estudiantes en ambas clases, el modelo Flipped Classroom permitió a los estudiantes una mayor independencia y autonomía del trabajo, pidieron la orientación de los profesores cuando precisaban, siendo ambos roles más lineales, de camaradería, que verticales, de jerarquía.

Y de acuerdo a la determinación de conocimiento de los estudiantes, se observa que aunque la diferencia no es ampliamente significativa, el Flipped Classroom refuerza el aprendizaje y construye nuevo conocimiento de manera más práctica. Pese a que no se analizó el nivel de satisfacción de los estudiantes, se observó que este modelo era más 
atractivo, ligeramente más productivo en conocimiento y mayormente enriquecedor en habilidades tanto del pensamiento como sociales.

Respecto al uso de las TIC en el aula, se concluye que con un manejo adecuado, es posible optimizar el tiempo de la clase, romper la rutina y favorecer el aprendizaje de los estudiantes. En este sentido, también se afirma que para que existan prácticas provechosas con las TIC, dependerá en gran medida de brindar a los profesores formación, no técnica, sino de experiencias de apropiación.

En tiempos donde es un hecho que existe una cultura digital que parece irreversible, se vuelve evidente la necesidad de implicar a los profesores a las experiencias con dichos ambientes, porque sólo en la medida en que vivencien esto, es como se sentirán capaces de explorar e incorporar modelos pedagógicos como el Flipped Classroom a sus salas de aula. Los profesores no se pueden seguir quedando atrás respecto al conocimiento de software y ambientes virtuales, porque esto los aleja de sus estudiantes e imposibilidad el diálogo con los mismos.

Por último, en palabras metafóricas de Bauman y apropiadas a este estudio, las TIC son como fluidos, "[...] no conservan una forma durante mucho tiempo y están constantemente dispuestos a cambiarla [...] a diferencia de los sólidos, no es posible detenerlos fácilmente" (BAUMAN, 2000, p. 8). Desde esa perspectiva, los estudiantes comparten las mismas cualidades. Sin embargo, muchas veces su formación es determinante para que en el desenvolvimiento de su profesión se tornen líquidos o sólidos, por eso la importancia de que el profesor consiga entender y dialogar con sus estudiantes el lenguaje de las TIC, para brindarles otras oportunidades de desenvolver habilidades que les permitan desplazarse con facilidad y en diferentes contextos, para así afrontar las complejas mudanzas de la contemporaneidad.

\section{Referencias}

BAUMAN, Zygmunt. Modernidad líquida. Buenos Aires: Grafinor, 2000.

BERGMANN, Jonathan; SAMS, Aaron. Flip your classroom: reach every student in every class every day. Oregon: Iste, 2012.

BERGMANN, Jonathan. Flipped-learning toolkit: overcoming common hurdles. Edutopia, San Raphael, nov. 4, 2014. Disponible en: <www.edutopia.org/blog/flipped-learning-toolkit-common-hurdles-jonbergmann>. Acceso en: 27 my. 2018.

BONILLA, Maria. Formação de professores em tempos de web 2.0. In: ASSUNÇÃO, MariaTeresa (Org.). Escola, tecnologias digitais e cinema. Juiz de Fora: UFJF, 2011. p. 59-87.

BONILLA, Maria; PRETTO, Nelson. Movimentos colaborativos, tecnologias digitais e educação: em aberto. Brasilia, DF: INEP, 2015.

CARTÓN, Ángel et al. Aprendizaje basado en problemas y flipped classroom: una experiencia de innovación docente en ingenierías del ámbito industrial. In: CONGRESO DE INNOVACIÓN EDUCATIVA EN LAS ENSEÑANZAS TÉCNICAS, 23., 2015, Valencia. Actas... Valencia: Universidad Politécnica de Valencia, 2015. p. 1-13. 
CASTELLS, Manuel. La era de la información: economía, sociedad y cultura. La sociedad red. Madrid: Alianza, 2000.

CRESWELL, John. Projeto de pesquisa: métodos qualitativo, quantitativo e misto. Porto Alegre: Artmed, 2007.

DAVIES, Randall; DEAN, Douglas; BALL, Nick. Flipping the classroom and instructional technology integration in a college-level information systems spreadsheet course. Educational Technology Research and Development, v. 4, n. 61, p. 563-580, agto. 2013.

FLICK, Uwe. Métodos de pesquisa: introdução à pesquisa qualitativa. Porto Alegre: Artmed, 2009.

GALEFFI, Dante. Didática filosófica mínima: ética do fazer-aprender a pensar de modo próprio e apropriado como educar transdisciplinar. Salvador: Quarteto, 2017.

HIRSCH, Joe. 4 tips for flipped learning. Edutopia, San Raphael, 2014. Disponible en: <www.edutopia.org/ blog/4-tips-for-flipped-learning-joe-hirsch>. Acceso en: 2 jun. 2018.

KINCHELOE, Joe. Rigour and complexity in educational research maintain: introduction the power of the bricolage: expanding research methods. London: Open University Press, 2004. Disponible en: <www. mheducation.co.uk/openup/chapters/0335214002.pdf>. Acceso en: 1 jun. 2018.

LEÓN, Orfelio; MONTERO, Ignacio. Diseño de investigaciones: introducción a la lógica de la investigación en psicología y educación. Madrid: McGraw Hill, 2003.

MASON, Greg; SHUMAN, Teodora; COOK, Kathleen. Comparing the effectiveness of an inverted classroom to a traditional classroom in an upper-division engineering course. Education IEEE Transactions, v. 1, n. 1, p. 430-435, nov. 2013.

MIGUEL-DÍAZ, Mario. Modalidades de enseñanza centradas en el desarrollo de competencias: orientaciones para promover el cambio metodológico en el marco del espacio europeo de educación superior. Oviedo: Universidad de Oviedo, 2005.

PRETTO, Nelson. Uma escola sem/com futuro. Salvador: Edufba, 2013.

SAMPAIO-S, Joseilda; BONILLA, Maria Helena. A cultura digital na formação de professores. Revista Tempos e Espaços em Educação, Aracajú, v. 7, n. 14, p. 23-34, 2014.

TOFFLER, Alvin. A terceira onda. Rio de Janeiro: Record, 1980.

WERTSCH, James. Vigotsky y la formación social de la mente. Madrid: Paidós, 1988.

Recibido en: 17.09.2018

Revisado en: 26.02.2019

Aprobado en: 09.04.2019 
Alvaro Adriazola Uribe es doctorando en Difusión del Conocimiento, Universidad Federal de Bahía (UFBA), Brasil. Máster en Ciencia Animal (2017), UFBA. Licenciado en Medicina Veterinaria (2012), Universidad Católica de Temuco, Chile.

Georgina Duran Jimenez es doctoranda en Educación, Universidad Federal de Bahía (UFBA). Máster en Investigación e Innovación en Currículum y Formación (2014), Universidad de Granada, España. Licenciada en Ciencias de la Comunicación (2010) y en Educación Física, Deporte y Recreación (2013), Universidad Veracruzana, México.

Marcelo Flores Troncoso es máster en Ciencias Veterinarias (2009), Universidad Austral de Chile, Chile. Licenciado en Medicina Veterinaria (1998), Universidad Austral de Chile, Chile. 